\section{Parathyroid carcinoma discovered in young trauma victim during intensive care management}

\author{
LJUBICA PEJAKOV • VESNA ČEJOVIĆ • ALEKSANDAR FILIPOVIĆ • \\ TANJA NENEZIĆ
}

LJUBICA PEJAKOV $(\square)$

Clinical Centre of Montenegro Clinic for anaesthesia intensive care and pain therapy Ljubljanska bb 81000 Podgorica, Montenegro Phone: ++38220225117

Fax: ++38220225284 E-mail: ljpejakov@gmail.com

VESNA ČEJOVIĆ Clinical Centre of Montenegro Clinic for anaesthesia intensive care and pain therapy

ALEKSANDAR FILIPOVIĆ Clinical Centre of Montenegro Clinic for surgery

TANJA NENEZIĆ

Clinical Centre of Montenegro Institute for Pathology

\begin{abstract}
Parathyroid gland carcinoma is uncommon disease, accounting less than $1 \%$ among the cases of primary hyperparathyroidism. There has been reported variations in prevalence between the nations, with higher occurence in Japan and Italy, according to their referral centers. Parathyroid cancer occurs mostly in patients above 30 years, but no sex differences have been noticed. Clinical signs correspond to symptoms of hypercalcaemia, which is usually severe, due to high production of parathyroid hormone in mostly functional tumors. Palpable mass in the neck and osteoporosis are present in about $50 \%$ of the cases.

We report a case of 24-year old female victim of traffic accident, suffering severe traumatic injuries- intracerebral and brainstem bleeding and contusions, together with femoral fracture, in which intensive care management simptoms suspicious to parathyroid carcinoma have been observed. Further imaging, laboratory findings, and clinical signs confirmed diagnosis. Surgery, with "en bloc" excision of the node together with adherent tissue has been performed. Definite histopathology finding approved parathyroid carcinoma. Clinical signs and laboratory findings normalized in the next period, and patient was discharged from our intensive care.

This case indicates that close monitoring of clinical signs, biochemical findings together with imaging modalities available, help not to miss such rare diseases, which might be masked by primary trauma.
\end{abstract}

Key words: polytrauma, parathyroid gland, carcinoma, treatment

\section{Introduction}

Parathyroid carcinoma is uncommon malignancy of the parathyroid glands, accounting less than $1 \%$ among the cases of primary hyperparathyroidism, although there are variations between the nations. $(1,2)$ We report a case of parathyroid carcinoma discovered in young polytraumatized patient.

\section{Case report}

A 24- year old female traffic accident trauma victim was admitted from distant regional hospital where intubated, to tertiary- level intensive care, seven hours after the event. On arrival at our intensive care unit, patient unconscious, Glasgow Coma Scale (GCS) scored 4 , febrile $40^{\circ} \mathrm{C}$, hypoventilating with irregular spontaneous breathing pattern, pupils symmetrical, midpositioned but small ( 2mm in diameter). On physical examination sinus tachycardia with 
pulse rate $180 \mathrm{bpm}$ and arterial blood pressure 100/60 mmHg. Motor responses showed flexor posturing. APACHE II score on admission was 29.

Computed tomography (CT) scan of the head revealed intracerebral haematomas located around and in the right ventricle, in the frontal-temporal region and in the diencephalon. Neuro-radiologist described contusions in the both parietal lobe and left frontal cerebral region, as well as in the brain stem (figure 1). Open fracture of the left femur with dislocation of the bone was managed at once, by orthopaedic surgeon, with stabilisation and longitudinal traction. Mechanical breathing assistance was instituted immediately. Neurosurgeon has decided for nonoperative treatment of the brain.

Laboratory findings indicate anaemia, leucocytosis and slightly elevated serum calcium level of $2.68 \mathrm{mmol} / \mathrm{L}$ (normal ranges 2.10-2.55 mmol/L). On the day 4 hypertension $(180 / 90 \mathrm{mmHg}$ ) occurred. Percutaneous tracheostomy and definite orthopaedic operation (reposition and osteosynthesis of the left femur), were performed under general anaesthesia, on the day 6 . Physiotherapy started three days after, but painful grimacing and spastic lower extremities were present.

Due to the presence of palpable mass in the right side of the neck, and clinical suspicion for thyroid gland function abnormality, imaging and hormonal examinations were done. Ultrasonography showed normal thyroid gland size, but under the right lobe heterogeneous nodal structure of about $20 \mathrm{~mm}$ in diameter was detected, partly cystic altered, without enlarged regional lymph node. The same was approved by contrast CT scan of the neck, which has been done shortly after. Scintigraphy was described as suspicion to nodular mass, probably belonging to right thyroid lobe.

High serum level of intact parathyroid hormone (iPTH) (Immulite ${ }^{\circledR} 2000$ Systems Analyzers), 46.0 pmol/L (normal ranges 1.30-6.80 pmol/L) was found. At the same time, serum calcium level started to increase around

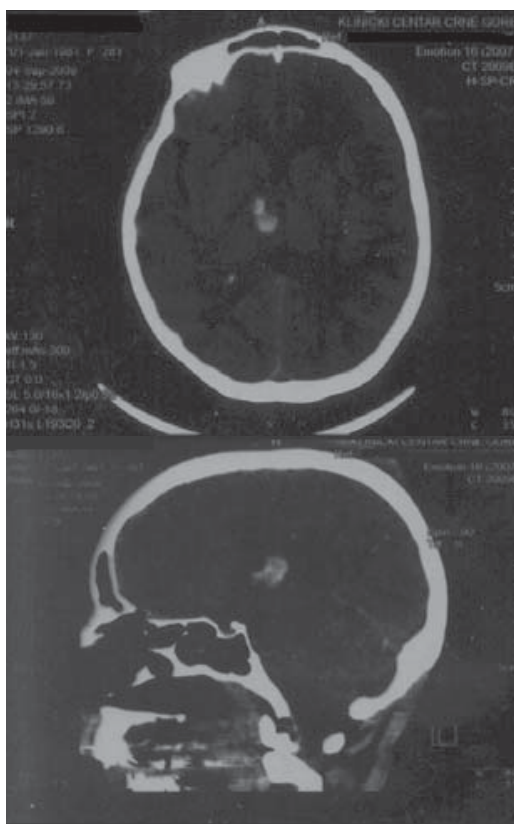

Figure 1. Computed tomography of the head at the admission.

$3.40 \mathrm{mmol} / \mathrm{L}$, while other hormones and electrolyte levels were normal. Urine calcium excretion, was increased, $24.36 \mathrm{mmol} /$ day (normal ranges 2,5-7,5 $\mathrm{mmol} /$ day). Calcitonin medication was advised by endocrinologist in order to lower hypercalcaemia. Osteodensitometry (Hologic ${ }^{\circledR}$ ) revealed osteopenia at the right hip with increased fracture risk.

As GCS rose to 9, and head CT scan control verified resorption of the intracerebral haematomas with normal ventricles and extracerebral liquor space, analgesia/sedation was excluded and percutaneous feeding gastrostomy was performed. Mode of mechanical ventilation changed to more spontaneous breathing.

After one month, serum calcium level was markedly elevated (4.55 mmol/L), phosphorus $2.40 \mathrm{mmol} / \mathrm{L}$ (range 0.80 $1.60 \mathrm{mmol} / \mathrm{L}$ ) and $\mathrm{iPTH} 58.3 \mathrm{pmol} / \mathrm{L}$. At that time control bronchoscopy finding was normal with sufficient spontaneous breathing. Despite of cardiologist's advices for therapy, heart rate still remained about 120 beat/minutes with high blood pressure (160/100 mm Hg).

Based on clinical signs, imaging results and biochemical parameters, endo-

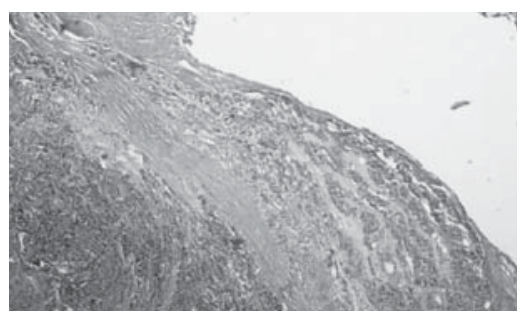

Figure 2. Hystopathology of the gland. Microscopic finding has been described "as tumorous tissue with solid areas and trabecula polygonal-main cells and oxyphil cells with eosinophil cytoplasm, that are hyper chromatic and with irregular nucleus. Very rare mitoses are present. Between tumour cells there are fibrotic septae without nucleus, which infiltrate groups of tumour cells. Tumour growth is infiltrative. No signs of angioinvasion on the border of the tumour. There is thyroid gland tissue peripherally, which is not infiltrated with tumourous tissue."

crinology surgeon decided to perform operative procedure, right sided parathyroidectomy. Node $23 \times 13 \mathrm{~mm}$ was removed through "en bloc" excision together with adherent tissue; "ex tempore» biopsy and later definite histopathology finding confirmed parathyroid carcinoma (figure 2).

During the first week after surgery serum calcium level decreases to the lowest value of $2.02 \mathrm{mmol} / \mathrm{L}$, while first iPTH control fall to $0,87 \mathrm{pmol} / \mathrm{L}$, although it fully normalized later.

One week postoperatively, clinical signs were normal; GCS scored 13, and before discharging, oncologist and endocrinologist ordered further imaging and laboratory controls at patient's regional hospital.

\section{Discussion}

The etiology of parathyroid carcinoma is mostly unknown, but association with previous radiation to neck and several genetic defects have been reported. (3) They usually occur in patients older than 30 years. $(1,4,5)$ Most are secreting parathyroid hormone, with abrupt and severe symptoms onset as a result of hipercalcaemia, which is seen in about two thirds of the patients. 
Serum calcium level is often above $3.6 \mathrm{mmol} / \mathrm{L}$ and refractory to medical treatment. (6) Elevated levels of serum iPTH are diagnostic and very high: 3-10 times above the upper limit of normal for the assay employed. (6) Anemia is also more common in carcinoma than in benign form. Palpable mass in the neck are present in about $50 \%$ of patients with parathyroidocarcinoma, as it was in our patient, too. $(1,6)$ In our case, parathyroid carcinoma was found out in younger patient; both, IPTH and calcium levels were typically elevated, but anemia was considered as a consequence of bleeding, not as a sign of carcinoma only.

Shaha and Shah (7) have proposed a staging system, which might help in uniform reporting of the extent of dise- ase in patients with parathyroid carcinoma. According to this classification, tumour in our patient was classified as stage I: T1N0M0, that means primary tumour $3 \mathrm{~cm}$, without regional lymph node metastases and no evidence of distant metastases.

Early parathyroidectomy is paramount for optimal outcome; indications are typical clinical symptoms of hypercalcaemia, calcium level > 2.9-3.0 mmol/l, urine calcium level $>10 \mathrm{mmol} / \mathrm{day}$, renal stones, reduced creatinine clearence $>30 \%$, age $<50$ years, osteoporosis and patient request. When the gross pathological findings suggest malignancy, «en bloc» resection of the tumor is mandatory, providing 90\% of the patients being long-term survivors. $(1,6,8)$
As no preoperative test could reliably distinguish parathyroid cancer from benign primary hyperparathyroidism, diagnosis is still based on the histological apperance of the excised parathyroid gland and clinical indicators. (9) Hystopatologically, the best predictors of malignancy are presence of invasion, fibrotic capsule with fibrous septa extending into the gland, and nuclear atypia. (10)

\section{Conclusion}

Parathyroid gland carcinoma is a very rare disease, which symtoms could be masked, especially in trauma victim patients. Close laboratory and clinical monitoring are the most important for prompt recognition and adequate treatmant.

\section{REFERENCES}

1. Fraker DL. Parathyroid tumors. In: DeVita VT, Jr, Hellman S, Rosenberg Sa, editors. Cancer: Principles and practice of oncology. 6th Ed. Philadelphia: Lippincott Williams and Wilkins; 2001; p. 1763-9.

2. Mittendorf EA, McHenry CR. Parathyroid carcinoma. J Surg Oncol 2005; 89(3):136-42.

3. Shattuck TM, Valimaki S, Obara T. Somatic and germ-line mutations of the HRPT2 gene in sporadic parathyroid carcinoma. N Engl J Med 2003; 349(18):1722-9

4. Marx SJ. Hyperparathyroid and hypoparathyroid disorders. N Engl J Med 2000;343:1863-75.

5. Hundahl SA, Fleming ID, Fremgen AM, Menck HR. Two hundred eighty-six cases of parathyroid carcinoma treated in the U.S. between 1985-1995: a National Cancer Data Base Report. The American College of Surgeons Commission on Cancer and the American Cancer Society. Cancer 1999;86(3):538-44.

6. Fraker DL. Update on the management of parathyroid tumors. Curr Opin Oncol 2000;12:41-8.

7. Shaha AR, Shah JP. Parathyroid carcinoma. A diagnostic and therapeutic challenge. Cancer 1999;86:378-80.

8. Grant CS, Thompson G. Primary hyperparathyroidism surgical management since the introduction of the minimally invasive parathyroidectomy. Mayo Clinic Experience. Arch Surg 2005;140:472-9

9. Shane E. Parathyroid carcinoma. J Clin Endocrinol Metab 2001;86:485-93.

10. Bondeson L, Sandelin K, Grimelius L. Histopathological variables and DNA cytometry in parathyroid carcinoma. Am J Surg Pathol 1993;17:820-9. 